\title{
A case of refractory multiple myeloma with proliferation of large granular lymphocytes by lenalidomide treatment and its association with clinical efficacy
}

\author{
MICHITOSHI HASHIGUCHI ${ }^{1,2}$, TAKASHI OKAMURA ${ }^{1}$, KEI NOMURA ${ }^{1}$, TAKAYUKI NAKAMURA $^{1}$, \\ KUNIKI KAWAGUCHI $^{1}$, SATOKO KOTEDA ${ }^{1}$, SATOSHI MORISHIGE ${ }^{1}$, EIJIROU OKU ${ }^{1}$, \\ YUKA TAKATA $^{1}$, RITSUKO SEKI ${ }^{1}$, FUMIHIKO MOURI ${ }^{1}$, KOICHI OSAKI $^{1}$, \\ KOHJI YOSHIMOTO $^{1}$, YUTAKA IMAMURA ${ }^{2}$ and KOJI NAGAFUJI ${ }^{1}$
}

\author{
${ }^{1}$ Division of Hematology and Oncology, Department of Internal Medicine, Kurume University School of Medicine, \\ Kurume, Fukuoka 830-0011; ${ }^{2}$ Department of Hematology, St. Mary's Hospital, Kurume, Fukuoka 830-8543, Japan
}

Received August 31, 2015; Accepted January 13, 2016

DOI: $10.3892 / \mathrm{mco} .2016 .747$

\begin{abstract}
A 72-year-old Japanese male was diagnosed as having monoclonal gammopathy of undetermined significance and was followed up without therapy. Three years later, the patient progressed to symptomatic multiple myeloma. Melphalan + prednisolone was administered as first-line chemotherapy for $\sim 6$ years. Since the patient was judged to exhibit refractory multiple myeloma, he subsequently received radiation therapy on the lumbar spine. The patient was enrolled in a clinical trial and received lenalidomide + lowdose dexamethasone (Rd) therapy. The patient achieved very good partial remission following four cycles of Rd. At this time, large granular lymphocytes (LGLs) increased to $25-40 \%$ of peripheral blood leukocytes, however, the LGLs were present in the blood ( 8\%) prior to lenalidomide treatment. By flow cytometry of surface antigens, it was revealed that the LGLs were positive for cluster of differnetiation (CD)2, 7, 8, 16, 56, and 57, and human leukocyte antigen-D related, however, were negative for $\mathrm{CD} 3,4$ and 5, suggesting that these LGLs predominantly exhibited an natural killer (NK) cell phenotype. T-cell receptor $\beta$ gene rearrangement was not detected by polymerase chain reaction. ${ }^{\mathrm{S}}{ }^{51} \mathrm{Cr}$ release assay was performed to investigate whether the NK cells actually possessed activity. A low level of $\mathrm{M}$ protein was sustained for $\sim 15$ months. This implied the enhancement of immune activation during lenalidomide treatment. The present case study suggested that LGL cells induced by lenalidomide may contribute to long-term
\end{abstract}

Correspondence to: Dr Takashi Okamura, Division of Hematology and Oncology, Department of Internal Medicine, Kurume University School of Medicine, Kurume, Fukuoka 830-0011, Japan

E-mail: okamura@med.kurumeu.ac.jp

Key words: large granular lymphocytes, lenalidomide, multiple myeloma, immunomodulatory drugs, immunomodulatory function restraint of myeloma cells. This immune system component may contribute to disease control.

\section{Introduction}

Multiple myeloma (MM) is a plasma cell tumor, which has differentiated from B lymphocytes, and the disease is characterized by the production of monoclonal immunoglobulin (Ig). Previously, several novel drugs for the treatment of MM have been developed and the clinical outcome has significantly improved. Examples of these are bortezomib, a proteasome inhibitor, and the immunomodulatory drugs (e.g. thalidomide and lenalidomide). Lenalidomide, a derivative of thalidomide, has been demonstrated to induce immune responses, including cytokine production, and the activation of natural killer (NK) cells and T cells, to exert antitumor effects directly on MM cells by arresting the cell cycle, and to induce decreases of interleukin (IL)-6 and vascular endothelial growth factor levels in the microenvironment of the tumor (1). This has given rise to a beneficial clinical effect in patients with MM (2). In a clinical trial (MM-009/010), the time to progression and overall survival were significantly prolonged in patients treated with lenalidomide in combination with dexamethasone, compared with those with dexamethasone alone, and the clinical efficacy was also confirmed (3). In earlier clinical studies, lenalidomide monotherapy was revealed to be useful as maintenance therapy for elderly patients (4) or post-transplant therapy $(5,6)$. Although it is hypothesized that lenalidomide therapy activates immune cells (7) and leads to good clinical efficacy. Limited data to show the direct correlation between the increase of immune cells and the clinical effects of lenalidomide in MM patients is currently available.

The present study reported a case of refractory MM with the emergence of large granular lymphocytes (LGLs) during lenalidomide treatment. Lenalidomide revealed efficacy to suppress serum M-protein level and tumor size in this case. This suggested a direct correlation between the proliferation of LGLs and the therapeutic effect of lenalidomide. 


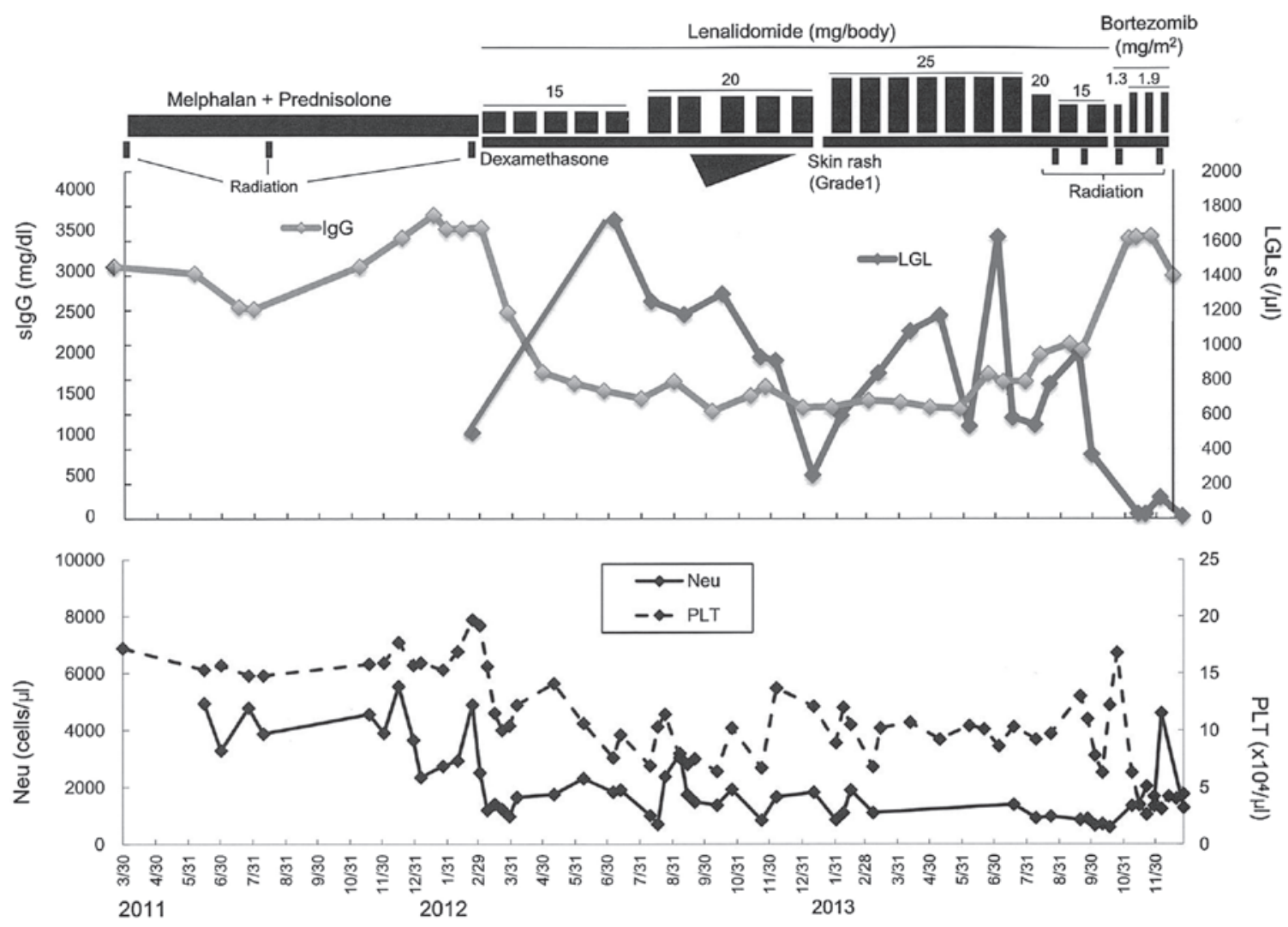

Figure 1. Clinical course of the present case. An inverse correlation was observed between the serum IgG and LGL levels. MP was administered as first-line chemotherapy. The patient subsequently received radiation therapy on the lumbar spine. As the disease activity had failed to be controlled by MP and radiation therapy, the patient was enrolled in a clinical trial and received Rd therapy. A low level of IgG was sustained during Rd therapy for $\sim 15$ months, associated with the increase of LGLs in peripheral blood. As extramedullary tumor was observed 15 months following the initiation of Rd therapy, BD therapy was started in addition to local radiation therapy. Notably, the number of LGLs decreased and serum IgG level was increased following BD treatment and radiation. MP, melphalan + prednisolone; Rd, lenalidomide + low-dose dexamethasone; BD, bortezomib + dexamethasone: LGL, large granular lymphocyte; sIgG; serum immunoglobulin G; Neu, neutrophil; PLT, platelet.

\section{Case report}

A 72-year-old Japanese male patient consulted a doctor for constipation. Laboratory examination revealed a high serum IgG level (3,305 mg/dl) with IgG к-type M-protein. However, aspiration analysis of bone marrow cells revealed no increase of plasma cells. The patient was diagnosed with monoclonal gammopathy of undetermined significance and was followed up without therapy. Three years later, the patient progressed to symptomatic MM. Subsequently, melphalan + prednisolone (MP) was administered as first-line chemotherapy. Following MP chemotherapy for $\sim 6$ years, the patient complained of lower back pain and was revealed by CT examination to exhibit vertebral mass lesions. The patient was subsequently referred to St. Mary's Hospital (Fukuoka, Japan). Since the patient was judged to exhibit refractory MM, he subsequently received radiation therapy on the lumbar spine. Serum $\operatorname{IgG}$ levels were elevated to $3,048 \mathrm{mg} / \mathrm{dl}$, while the proportion of myeloma cells was $15.2 \%$ in the bone marrow. No chromosomal abnormalities were detected. Durie-Salmon stage classification was IIA and International Staging System classification was I. As the disease activity failed to be controlled by MP and radiation therapy, the patient was enrolled in a clinical trial and received lenalidomide (15 mg, days 1-21 of a 28-day cycle) + low-dose dexamethasone (20 mg/week) (Rd) therapy. This clinical study was designed to investigate the maximum optimal dosage and efficacy of lenalidomide (UMIN Clinical Trials Registry number, UMIN000010178), and treatment by the administration of lenalidomide + dexamethasone was initiated (Fig. 1).

Serum $\mathrm{IgG}$ gradually decreased to $1,530 \mathrm{mg} / \mathrm{dl}$ and reduction of the size of the bone tumors was observed (Fig. 2A and B). At this time, LGLs, which were defined as being more than double the size of red blood cell with abundant cytoplasm containing azurophilic granules (Fig. 3A), increased to $25-40 \%$ of peripheral blood leukocytes, although the LGLs were present in blood $(\sim 8 \%)$ prior to lenalidomide treatment. The patient achieved very good partial regression following four cycles of $\mathrm{Rd}$ therapy. In terms of the adverse effect of lenalidomide, skin rash (Grade 1) appeared; however, it subsided spontaneously upon the continuation of lenalidomide. A low level of $\operatorname{IgG}$ was sustained for $\sim 15$ months. Flow cytometry of surface antigens revealed that LGLs exhibited positivity for cluster of differentiation (CD)2, 7, 8, 16, 56 and 57, and human leukocyte antigen-D related, however, were negative for $\mathrm{CD} 3,4$, and 5(Fig. 3B), suggesting that these LGLs predominantly exhibited an natural killer (NK) cell phenotype. T-cell receptor $\beta$ gene rearrangement was not detected by polymerase chain reaction. $\mathrm{A}^{51} \mathrm{Cr}$ release assay was performed to investigate whether the NK cells actually possessed activity. The cytotoxic activity was revealed to be elevated to $60 \%$ (normal range, $18-40 \%$ ). 
A

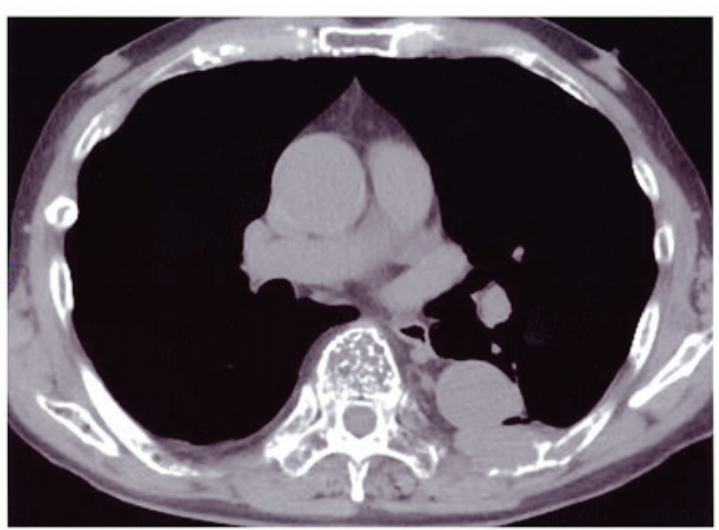

B

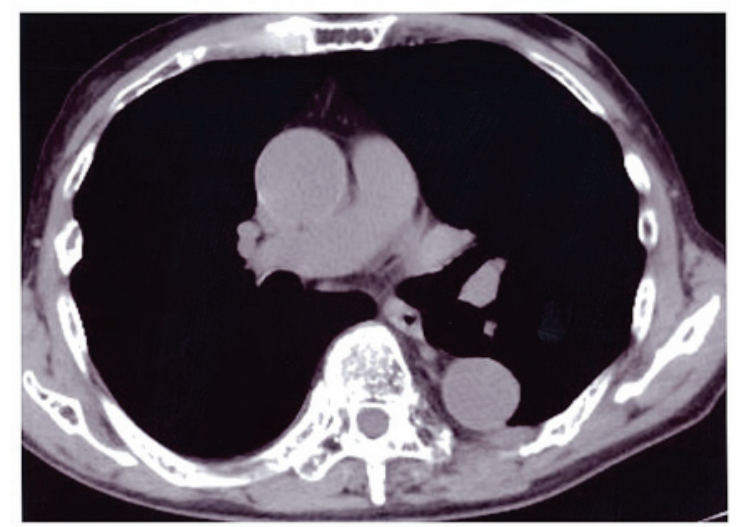

Figure 2. CT imaging. (A) Following melphalan + prednisolone chemotherapy for $\sim 6$ years, the patient exhibited bone tumor lesions, as determined by CT examination (1 March 2012). (B) When serum immunoglobulin $\mathrm{G}$ gradually decreased to $1,530 \mathrm{mg} / \mathrm{dl}$, reduction of the size of the bone tumors was observed (19 July 2013). CT, computed tomography.

A

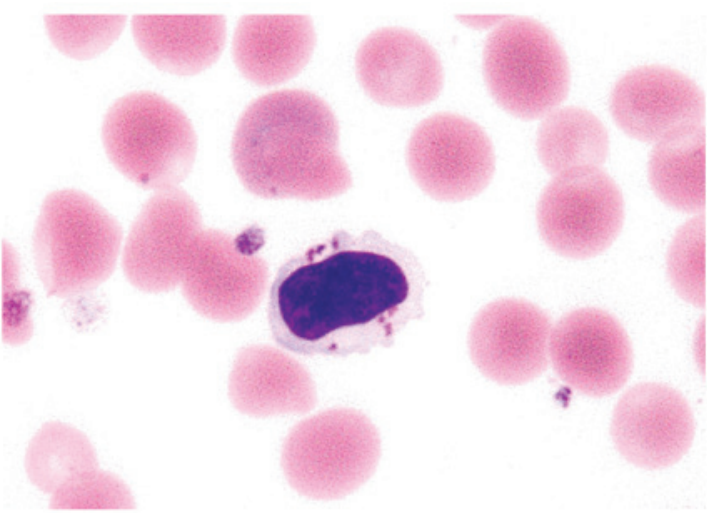

B

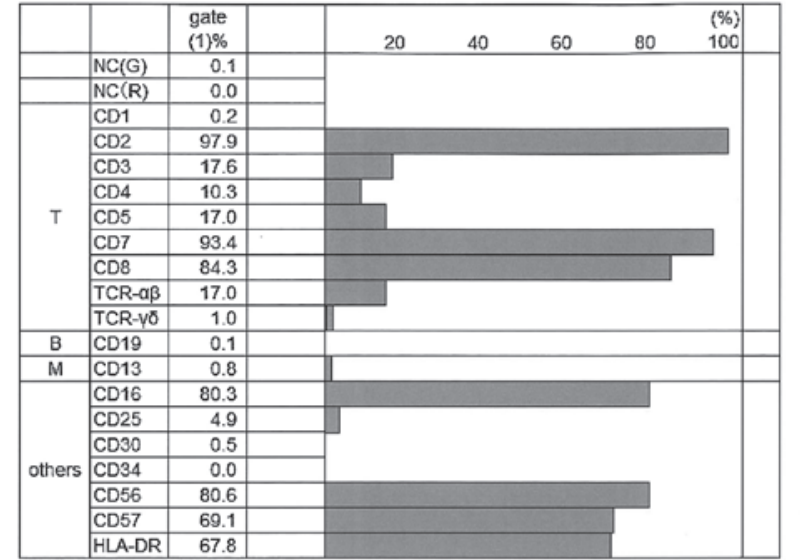

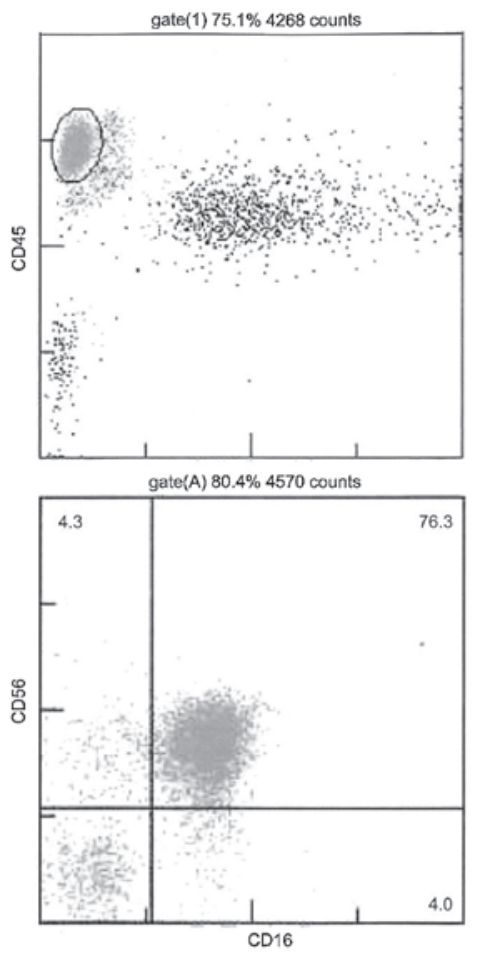

Figure 3. Morphology and immunophenotype of LGLs. (A) When the patient achieved VGPR after four cycles of lenalidomide + low-dose dexamethasone LGLs, which was defineed as more than double the size of red blood cell and abundant cytoplasm, containing azurophilic granules, increased to $25-40 \%$ of peripheral blood leukocytes. (May-Giemsa staining; magnification, x400). (B) Flow cytometry of surface antigens revealed that LGLs were positive for CD2, 7, 8, 16, 56 and 57 and HLA-DR, however, were negative for CD3, 4, and 5, suggesting that these LGLs exhibited an NK cell phenotype. LGL, large granular lymphocyte; CD, cluster of differentiation; NK, natural killer; VGPR, very good partial remission; HLA-DR, human leukocyte antigen-D related; TCR, T cell receptor; $\mathrm{NC}$, negative control. 
Since an extramedullary tumor was observed 15 months following the initiation of lenalidomide therapy, bortezomib + dexamethasone (BD) therapy was initiated in addition to local radiation therapy. Unfortunately, exposure to this treatment was not effective for this patient. Notably, the number of LGLs decreased and serum IgG level was elevated following BD treatment and radiation. As shown in Fig. 1, an inverse correlation between the serum IgG and LGL levels was observed.

\section{Discussion}

The present case study reported an interesting case of refractory MM presenting the proliferation of LGLs in peripheral blood during lenalidomide therapy. These LGLs exhibited an NK cell phenotype $\left(\mathrm{CD} 16^{+} \mathrm{CD} 56^{+} \mathrm{CD} 57^{+}\right)$and cytotoxic activity, and may be involved in the elimination of myeloma cells. In the present case, a long-term decrease in M-proteins may be achieved by LGL expansion through lenalidomide treatment.

Induction of LGL cells is well known in chronic myeloid leukemia patients treated with dasatinib, which acts to eliminate BCR/ABL-positive cells and is associated with a favorable prognosis (8).

It has been reported that patients with relapsed/progressive MM exerted a marked deficit of NK/T cell numbers and activity, particularly following repeated chemotherapy and/or autologous peripheral blood stem cell transplantation following a high-dose melphalan regimen (2). Therefore, it appears to be beneficial for immunomodulatory therapy to increase the cytotoxic activity and cytokine production of NK/T cells in chemotherapy-resistant patients with MM.

The action of immunomodulatory drugs, including lenalidomide, is considered to be associated with cereblon (2). However, the detailed mechanisms remain to be elucidated. Lenalidomide normally induces immunomodulatory function when used alone. However, when used together with dexamethasone, the immunomodulatory effect is suppressed (9). In the current patient, although peripheral blood levels of IL-2 and interferon (IFN)- $\gamma$ were not elevated during lenalidomide treatment, and this may have been the result of the effect of combination with dexamethasone.

It was reported that CD57 is a marker of long-lived 'memory' NK cells (10). Notably, although the CD57 ${ }^{+} \mathrm{NK}$ cell population produced less IFN- $\gamma$ compared with CD57 cells in response to activation by cytokines, they exhibited higher levels of perforin and granzymes (10). In addition, it was also demonstrated that $\mathrm{CD} 7^{+} \mathrm{NK}$ cells are more sensitive to CD16 activation (10). Since lenalidomide can increase CD16 expression in NK cells (11), CD16 ${ }^{+} \mathrm{CD} 56^{+} \mathrm{CD} 57^{+}$cells, which were mentioned in this case report, may have antitumor potency under lenalidomide treatment. Previously, it was reported that no change was observed in $\mathrm{CD} 57^{+} \mathrm{NK}$ cell numbers following three cycles of Rd treatment (12), whereas the present study detected LGL expansion during the fifth cycle of Rd treatment. Therefore, these cells may not proliferate in the early phase of $\mathrm{Rd}$ treatment.

Since anti-myeloma therapy was not effective during BD treatment and LGL level was decreased, NK cell-mediated cytotoxic activity was suggested not to be sustained in this period. The MM cells may result in escape from the immune surveillance system through alteration in the cellular and/or extracellular properties during treatment (13). Certain heterogeneous tumor subclones with low immunogenicity can have a chance to survive and expand (14). In addition, MM cells are not fixed on a single evolutionary trajectory and can potentially shift between stable and evolving genomes during the course of the disease (15). In addition, epigenetic silencing of tumor antigen expression may be responsible for antigen loss and tumor escape (16). In the present case, MM cells may have escaped from immune surveillance during treatment.

In conclusion, the present case study demonstrated the proliferation of LGLs during lenalidomide + dexamethasone therapy, and demonstrated that the expanded LGLs were of the $\mathrm{CD} 57^{+} \mathrm{NK}$ lineage. This immune system component may contribute to disease control.

The accumulation of cases would provide more marked evidence of the beneficial effects of LGL in lenalidomide therapy. However, further investigation is required to reveal the precise mechanism of the correlation between clinical effects and LGLs during lenalidomide treatment.

\section{References}

1. Quach H, Ritchie D, Stewart AK, Neeson P, Harrison S, Smyth MJ and Prince HM: Mechanism of action of immunomodulatory drugs (IMiDS) in multiple myeloma. Leukemia 24: 22-32, 2010.

2. Chan AC, Neeson P, Leeansyah E, Tainton K, Quach H, Prince HM, Harrison SJ, Godfrey DI, Ritchie D and Berzins SP: Natural killer T cell defects in multiple myeloma and the impact of lenalidomide therapy. Clin Exp Immunol 175: 49-58, 2014.

3. Dimopoulos MA, Chen C, Spencer A, Niesvizky R, Attal M, Stadtmauer EA, Petrucci MT, Yu Z, Olesnyckyj M, Zeldis JB, et al: Long-term follow-up on overall survival from the MM-009 and MM-010 phase III trials of lenalidomide plus dexamethasone in patients with relapsed or refractory multiple myeloma. Leukemia 23: 2147-2152, 2009.

4. Palumbo A, Hajek R, Delforge M, Kropff M, Petrucci MT, Catalano J, Gisslinger H, Wiktor-Jędrzejczak W, Zodelava M, Weisel K, et al: Continuous lenalidomide treatment for newly diagnosed multiple myeloma. N Engl J Med 366: 1759-1769, 2012.

5. Attal M, Lauwers-Cances V, Marit G, Caillot D, Moreau P, Facon T, Stoppa AM, Hulin C, Benboubker L, Garderet L, et al: Lenalidomide maintenance after stem-cell transplantation for multiple myeloma. N Engl J Med 366: 1782-1791, 2012.

6. McCarthy PL, Owzar K, Hofmeister CC, Hurd DD, Hassoun H, Richardson PG, Giralt S, Stadtmauer EA, Weisdorf DJ, Vij R, et al: Lenalidomide after stem-cell transplantation for multiple myeloma. N Engl J Med 366: 1770-1781, 2012.

7. Neuber B, Herth I, Tolliver C, Schoenland S, Hegenbart U, Hose D, Witzens-Harig M, Ho AD, Goldschmidt H, Klein B and Hundemer M: Lenalidomide enhances antigen-specific activity and decreases CD45RA expression of T cells from patients with multiple myeloma. J Immunol 187: 1047-1056, 2011.

8. MustjokiS,Ekblom M, Arstila TP,Dybedal I,Epling-Burnette PK, Guilhot F, Hjorth-Hansen H, Höglund M, Kovanen P, Laurinolli T, et al: Clonal expansion of T/NK-cells during tyrosine kinase inhibitor dasatinib therapy. Leukemia 23: 1398-1405, 2009.

9. Gandhi AK, Kang J, Capone L, Parton A, Wu L, Zhang LH, Mendy D, Lopez-Girona A, Tran T, Sapinoso L, et al: Dexamethasone synergizes with lenalidomide to inhibit multiple myeloma tumor growth, but reduces lenalidomide-induced immunomodulation of T and NK cell function. Curr Cancer Drug Targets 10: 155-167, 2010.

10. Lopez-Vergès S, Milush JM, Schwartz BS, Pando MJ, Jarjoura J, York VA, Houchins JP, Miller S, Kang SM, Norris PJ, et al: Expansion of a unique $\mathrm{CD} 57^{+} \mathrm{NKG} 2 \mathrm{Chi}$ natural killer cell subset during acute human cytomegalovirus infection. Proc Natl Acad Sci USA 108: 14725-14732, 2011.

11. Zhang L, Qian Z, Cai Z, Sun L, Wang H, Bartlett JB, Yi Q and Wang M: Synergistic antitumor effects of lenalidomide and rituximab on mantle cell lymphoma in vitro and in vivo. Am J Hematol 84: 553-559, 2009. 
12. Hsu AK, Gherardin N, Quach H, Harrison SJ, Prince HM, Ritchie D and Neeson P: CD57+ NK cells are increased in patients with multiple myeloma and are primed effectors for ADCC, but not natural cytotoxicity. Blood 122: 1904, 2013.

13. Katira P,Bonnecaze RT and Zaman MH: Modeling the mechanics of cancer: Effect of changes in cellular and extra-cellular mechanical properties. Front Oncol 3: 145, 2013.

14. Magrangeas F, Avet-Loiseau H, Gouraud W, Lodé L, Decaux O, Godmer P, Garderet L, Voillat L, Facon T, Stoppa AM, et al: Minor clone provides a reservoir for relapse in multiple myeloma. Leukemia 27: 473-481, 2013
15. Keats JJ, Chesi M, Egan JB, Garbitt VM, Palmer SE, Braggio E, Van Wier S, Blackburn PR, Baker AS, Dispenzieri A, et al: Clonal competition with alternating dominance in multiple myeloma. Blood 120: 1067-1076, 2012.

16. DuPage M, Mazumdar C, Schmidt LM, Cheung AF and Jacks T: Expression of tumour-specific antigens underlies cancer immunoediting. Nature 482: 405-409, 2012. 